\title{
Ongoing Clinical Trials in Prostate Cancer: The STAMPEDE Trial
}

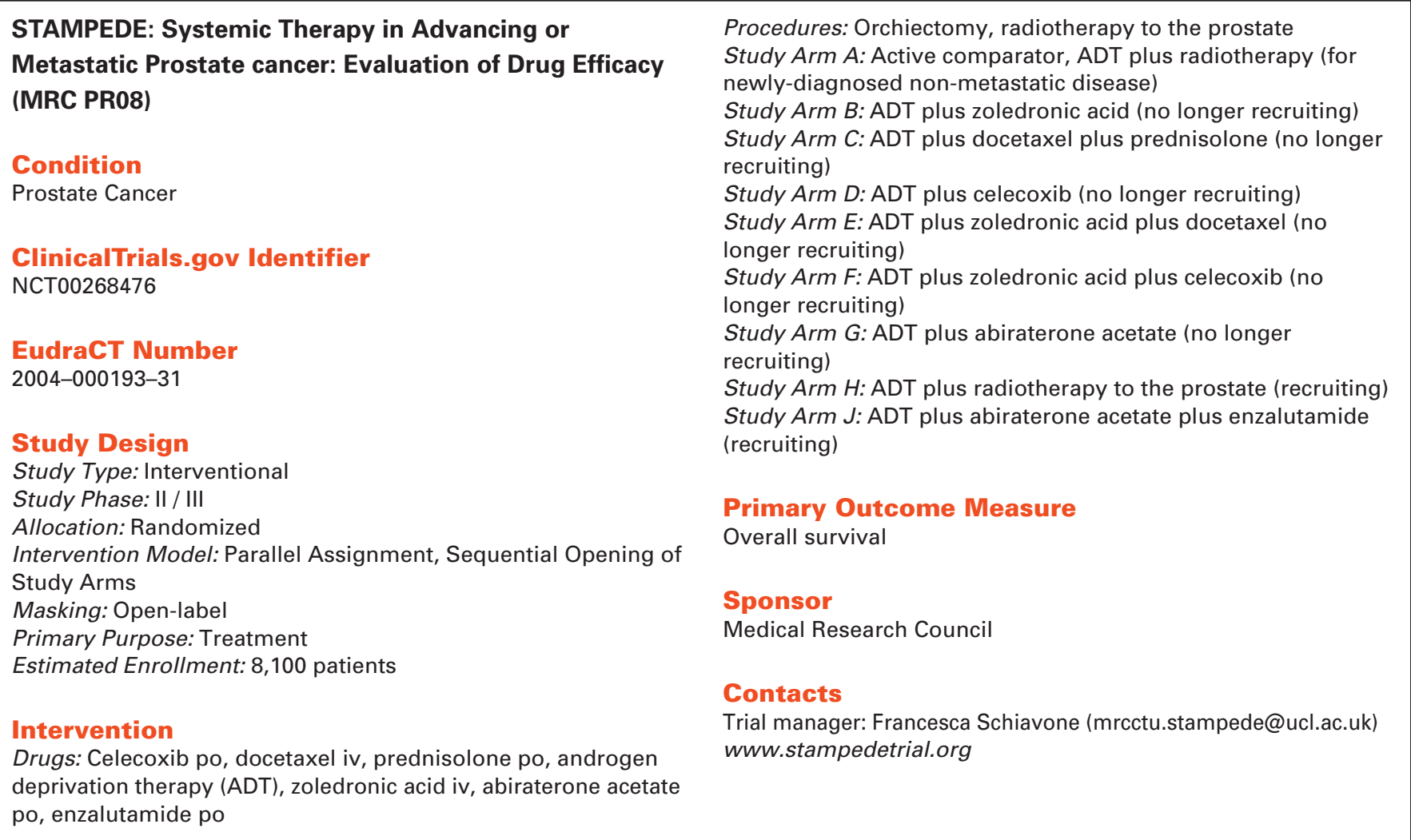

\section{Description}

This UK-led randomized, open-label trial has been initiated by the Medical Research Council and started recruitment in October 2005. Its large-scale multiarm, multistage, multicenter design is thought to compare the standard of care in hormone-naive prostate cancers, which is androgen deprivation therapy (ADT), to ADT in combination with 1 or 2 different systemic and / or local treatments, i.e. chemotherapy, bisphosphonates, androgen receptor signaling inhibitors, or radiotherapy. Patients included will have either newly diagnosed localized high-risk, node-positive or metastatic prostate cancers, as well as relapsed disease after previous radical prostatectomy or radiotherapy.

In this largest randomized trial in prostate cancer conducted to date, a total of 10 different study arms are planned to enroll 8,100 patients with an allocation ratio of 2 patients into the control arm (arm A) to 1 patient into one of the 9 experimental arms, of which 6 arms are no longer recruiting after inclusion of more than 7,000 patients. Currently, patients are randomized into one of the remaining 3 arms (arm A, H and J), study completion date for final data collection for primary outcome measure is estimated for September 2017.

Celecoxib (arm D) had shown antiproliferative effect against prostate cancer in vitro and has been evaluated as part of a phase II trial within the STAMPEDE trial. All other study arms are planned as a phase III trial. The primary outcome measure is overall survival (OS) with a targeted relative $25 \%$ OS improvement. Secondary study end points are failure-free survival, toxicity, quality of life, skeletal-related events, and cost effectiveness of the different treatments.

\begin{tabular}{|c|c|}
\hline KARGER & $\begin{array}{l}\text { (c) } 2016 \text { S. Karger GmbH, Freiburg } \\
2296-5270 / 16 / 0397-0464 \$ 39.50 / 0\end{array}$ \\
\hline Fax +497614520714 & \\
\hline $\begin{array}{l}\text { Information@Karger.com } \\
\text { www.karger.com }\end{array}$ & $\begin{array}{l}\text { Accessible online at: } \\
\text { www.karger.com/ort }\end{array}$ \\
\hline
\end{tabular}




\section{Eligibility Criteria}

\section{Inclusion Criteria}

Gender eligible for study: Male

High-risk newly diagnosed non-metastatic node-negative disease Both:

At least two of:

- Stage T3/4, PSA $\geq 40 \mathrm{ng} / \mathrm{ml}$, or Gleason sum score 8-10

- Intention to treat with radical radiotherapy (unless there is a contra-indication; exemption can sought in advance of consent, after discussion with MRC CTU)

OR newly diagnosed metastatic or node-positive disease At least one of:

- Stage Tany N+M0

- Stage Tany Nany M+

OR previously treated with radical surgery and/or radiotherapy, now relapsing

At least one of:

- PSA $\geq 4 \mathrm{ng} / \mathrm{ml}$ and rising with doubling time less than 6 months

- $P S A \geq 20 \mathrm{ng} / \mathrm{ml}$

- $\mathrm{N}+$

- $\mathrm{M}+$

\section{AND for all patients}

I. Histologically confirmed prostate adenocarcinoma

II. Intention to treat with long-term androgen deprivation therapy

III. Fit for all protocol treatment and follow-up, WHO performance status $0-23$

IV. Have completed the appropriate investigations prior to randomisation

$\mathrm{V}$. Adequate haematological function: neutrophil count $>1.5 \mathrm{x}$ 109/1 and platelets $>100 \times 109 / 1$

VI. Estimated creatinine clearance $>30 \mathrm{ml} / \mathrm{min}$

VII. Serum potassium $\geq 3.5 \mathrm{mmol} / \mathrm{L}$

VIII. Written informed consent

IX. Willing and expected to comply with follow-up schedule

$\mathrm{X}$. Using effective contraceptive method if applicable

\section{Exclusion Criteria}

Patients must not fulfil any of the criteria, below.

I. Prior systemic therapy for locally advanced or metastatic prostate cancer except as listed in section 4.1.3

II. Metastatic brain disease or leptomeningeal disease

III. Abnormal liver functions consisting of any of the following: Serum bilirubin $\geq 1.5 \times$ ULN (except for patients with Gilbert's disease, for whom the upper limit of serum bilirubin is $51.3 \mu \mathrm{mol} / \mathrm{l}$ or $3 \mathrm{mg} / \mathrm{dl}$ ). Aspartate aminotransferase (AST) or alanine aminotransferase $(\mathrm{ALT}) \geq 2.5 \times \mathrm{ULN}$

IV. Any other previous or current malignant disease which, in the judgement of the responsible physician, is likely to interfere with STAMPEDE treatment or assessment
V. Patients with contra-indications to prednisolone, including active peptic ulceration or a history of gastrointestinal bleeding

VI. Patients with active inflammatory bowel disease

VII. Symptomatic peripheral neuropathy grade (NCI CTC)

VIII. Any surgery (e.g. TURP) performed within the past 4 weeks

IX. Patients with significant cardiovascular disease such that, in the investigator's opinion, the patient is unfit for any of the study treatments. This might include: Severe/unstable angina, myocardial infarction less than 6 months prior to randomization, arterial thrombotic events less than 6 months prior to randomization, clinically significant cardiac failure requiring treatment (NYHA II-IV), cerebrovascular disease (e.g. stroke or transient ischaemic episode) less than 2 years prior to randomization, patients with uncontrolled hypertension defined as systolic BP greater or equal than $160 \mathrm{mmHg}$ or diastolic BP greater or equal than $95 \mathrm{mmHg}$.

$\mathrm{X}$. Patients receiving treatment with drugs known to induce CYP3A4 (including phenytoin, carbamazepine, Phenobarbital)

XI. Prior exposure to abiraterone

XII. Prior exposure to enzalutamide

XIII. Prior chemotherapy for prostate cancer

XIV. Prior therapy with zoledronic acid or other bisphosphonates other than treatment for hypercalcaemia or low bone density

XV. Prior exposure to policy of long-term hormone therapy before randomisation (unless as described in section 4.4.2)

XVI. History of seizure including any febrile seizure, loss of consciousness, or transient ischaemic attack within 12 months of randomisation or any condition that may pre-dispose to seizure (e.g., prior stroke, brain arteriovenous malformation, head trauma with loss of consciousness requiring hospitalization)

XVII. Unexplained history of loss of consciousness within 12 months of randomisation

XVIII. Operation of heavy machinery during treatment

Selection criteria for comparison of research $(m 1) R T$ for metastatic disease

All patients meeting criteria in section 4.1 and 4.2 are eligible for the trial, but not all can be allocated to the research (M1) radiotherapy arm. The selection criteria for this 'RT to the prostate' comparison are:

- Newly-diagnosed prostate cancer

- Demonstrable M1 disease

- No contraindication to radiotherapy e.g. no previous pelvic radiotherapy and no history of inflammatory bowel disease

- No previous radical prostatectomy

Any patients meeting these criteria will have a chance to be allocated to arm $\mathrm{H}$. 


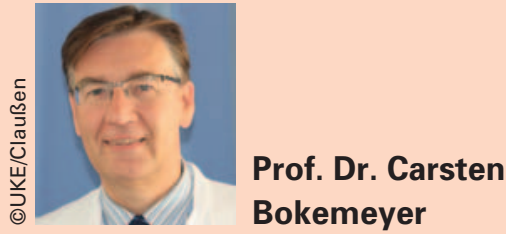

Why this Trial Is Important to Us

Prostate cancer is the most common malignancy and a leading cause of cancer deaths in men [1]. The growth-promoting properties of androgens on prostate cancer have been identified already in 1941, when bilateral orchiectomy led to significant shrinkage of prostate cancer metastases [2]. Since then, androgen deprivation therapy (ADT) has been standard of care for highrisk non-metastatic and also metastatic hormone-sensitive prostate cancer. Despite the generally good response to ADT, ADT alone is no curative treatment for most of the patients, but ADT in combination with radiotherapy (RT) has improved overall survival (OS) by $30 \%$ and a reduction of cancer-specific mortality by $54 \%$ in localized intermediate risk (NOM0) disease [3]. In locally advanced prostate cancer the addition of ADT + RT also beneficially impacted OS over ADT alone (hazard ratio (HR) 0.70) [4]. A post-hoc analysis of 721 patients with newly diagnosed M0 disease included into the STAMPEDE trial confirmed the beneficial use of ADT + RT for both N0M0 patients (HR 0.33 ), and for the first time for $\mathrm{N}+\mathrm{M} 0$ disease (HR 0.48) regarding failure-free survival (FFS) [5]. Moreover, a first interim analysis of the STAMPEDE trial did not show any survival benefit by adding the cyclooxygenase-inhibitor celecoxib to ADT (arm D) over ADT alone (control arm A) in newly diagnosed prostate cancer [6].

Until recently, docetaxel-based conventional chemotherapy was only offered to patients suffering from castration-resistant (after failure of ADT) locally advanced and / or metastatic prostate cancer. The GETUG-AFU 15 trial was the first randomized trial to favor the early use of docetaxel in combination with ADT $(\mathrm{ADT}+\mathrm{D})$ in hormone-naïve metastatic prostate cancer yielding a significantly improved radiographic (HR 0.75) and biochemical progression-free survival (HR 0.73), but the trial failed to show an OS benefit by $\mathrm{ADT}+\mathrm{D}$ as the primary endpoint [7]. Beyond that, the randomized CHAARTED trial reported a significant 13month OS improvement by ADT + D versus ADT in hormonenaïve metastatic prostate cancer ( 47.6 vs. 44.0 months; $\mathrm{p}<0.001$ ) [8]. The randomized phase III GETUG 12 trial was the first trial to report a beneficial impact of ADT $+\mathrm{D}$ in high-risk non-metastatic disease on relapse-free survival (HR 0.71; $\mathrm{p}=0.017$ ) [9]. These findings are in line with a first analysis of the larger STAMPEDE trial introduced here. In this analysis, ADT + D signifi-

\section{Dr. Christoph Oing}

cantly improved FFS for both metastatic (HR 0.62) and nonmetastatic patients (HR 0.57), while OS was only significantly improved in M1-disease (HR 0.73) (as it was reported by the CHAARTED trial). Adding zoledronic acid to hormone-sensitive disease did not impact FFS or OS, whereas addition of zoledronic acid to ADT $+\mathrm{D}$ improved survival but without a substantial benefit over ADT + D [10]. Follow-up of both the GETUG 12 and the STAMPEDE ADT + D study arms is yet insufficient for final estimation of the OS benefit. At last, the phase III RTOG 0521 trial reported a significant improvement of 4-year OS from 89 to $93 \%$ in high-risk localized disease by the addition of adjuvant docetaxel following ADT + RT [11].

The first analyses of the UK-led STAMPEDE trial, together with smaller phase III trials, yield essential data to substantially improve treatment and outcome of patients with hormone-naïve non-metastatic high-risk and metastatic prostate cancer. Addition of docetaxel should be considered in men with metastatic disease eligible for combined treatment, and even for selected men with high-risk non-metastatic disease, where at least FFS can be improved. The early use of zoledronic acid instead did not add any benefit, which is why bisphosphonates are only recommended for castration-resistant metastatic patients. In the future, STAMPEDE will also elucidate the potential of the early use of novel anti-androgenic treatment with abiraterone acetate alone (arm G; already closed) and together with enzalutamide (arm J), which to date have shown to be efficacious in castration-resistant metastatic disease before and after application of docetaxel, and for newly metastatic patients the combined application of ADT + radiotherapy + docetaxel $(\operatorname{arm} \mathrm{H})$. Moreover, the multistage, multiarm design appears feasible and efficient to answer complex clinical questions.

Summing up, the STAMPEDE trial has already reported first data of paramount importance, which will impact the treatment of hormone-senstitive high-risk localized and metastatic prostate cancer. Longer follow-up and analyses of the remaining study arms, particularly regarding the early use of the new anti-androgenic agents abiraterone acetate and enzalutamide holds the promise to answer highly relevant questions to substantially improve the standard of care for hormone-naïve prostate cancer patients. 


\section{References}

1 Attard G, et al., Prostate cancer. Lancet 2016;387:70-82.

2 Huggins C, Stevens RE Jr., Hodges CV: Studies on prostatic cancer II. The effects of castration on advanced carcinoma of the prostatic gland. Arch Surg 1941;43:209223.

3 Jones $\mathrm{CU}$, et al.: Radiotherapy and short-term androgen deprivation for localized prostate cancer. N Engl J Med 2011;365:107-118.

4 Mason MD, et al.: Final report of the intergroup randomized study of combined androgen-deprivation therapy plus radiotherapy versus androgen-deprivation therapy alone in locally advanced prostate cancer. J Clin Oncol 2015:33:2143-2150.

5 James ND, et al.: Failure-free survival and radiotherapy in patients with newly diagnosed nonmetastatic prostate cancer: data from patients in the control arm of the STAMPEDE trial. JAMA Oncol 2016;2:348-357.

6 James ND, et al.: Celecoxib plus hormone therapy versus hormone therapy alone for hormone-sensitive prostate cancer: first results from the STAMPEDE multiarm, multistage, randomised controlled trial. Lancet Oncol 2012;13:549-558.

7 Gravis G, et al.: Androgen deprivation therapy (ADT) plus docetaxel versus ADT alone in metastatic non castrate prostate cancer: impact of metastatic burden and long-term survival analysis of the randomized phase 3 GETUG-AFU15 trial. Eur Urol 2015; doi: 10.1016/j.eururo.2015.11.005.

8 Sweeney CJ, et al.: Chemohormonal therapy in metastatic hormone-sensitive prostate cancer. N Engl J Med 2015;373:737-746.
9 Fizazi K, et al.: Androgen deprivation therapy plus docetaxel and estramustine versus androgen deprivation therapy alone for high-risk localised prostate cancer (GETUG 12): a phase 3 randomised controlled trial. Lancet Oncol 2015;16:787-794.

10 James ND, et al.: Addition of docetaxel, zoledronic acid, or both to first-line longterm hormone therapy in prostate cancer (STAMPEDE): survival results from an adaptive, multiarm, multistage, platform randomised controlled trial. Lancet 2016; 387:1163-1177.

11 Sandler HM, et al.: A phase III protocol of androgen suppression (AS) and 3DCRT/ IMRT versus AS and 3DCRT/IMRT followed by chemotherapy (CT) with docetaxel and prednisone for localized, high-risk prostate cancer (RTOG 0521). J Clin Oncol 2015;33(suppl): abstr LBA5002.

\section{Contact address:}

Dr. Christoph Oing and Prof. Dr. Carsten Bokemeyer

Department of Oncology, Hematology and Bone Marrow Transplantation with Section of Pneumology University Cancer Center Hamburg (UCCH), University Medical Center Hamburg-Eppendorf Martinistraße 52, 20246 Hamburg, Germany

c.oing@uke.de 